\title{
Die Rolle der diabetischen Neuropathie bei der Genese des Charcot-Fußes
}

\section{Diabetic Neuropathy and the Charcot Foot}

\author{
Autoren \\ Maike F. Dohrn ${ }^{1}$, Sigurd Kessler ${ }^{2}$, Manuel Dafotakis ${ }^{1}$ \\ Institute \\ 1 Neurologische Klinik, Universitätsklinik der RWTH \\ Aachen, Aachen \\ 2 OTHOEVO, München \\ Schlüsselwörter \\ Charcot-Fuß, Diabetische Neuropathie, Neurogene \\ Inflammation, Knocheninnervation, Diabetes mellitus
}

Key words

Charcot foot, diabetic neuropathy, neurogenic inflammation, bone innervation, diabetes mellitus

Bibliografie

DOI https://doi.org/10.1055/a-1134-2547

Online-Publikation: 28.4.2020

Klin Neurophysiol 2020; 51: 67-72

(c) Georg Thieme Verlag KG Stuttgart · New York

ISSN 1434-0275

\author{
Korrespondenzadresse \\ PD Dr. Manuel Dafotakis \\ Neurologische Klinik \\ Universitätsklinikum Aachen \\ Pauwelsstraße 3352074 Aachen \\ mdafotakis@ukaachen.de
}

\section{ZUSAMMENFASSUNG}

Die neuropathische Osteo(arthro-)pathie, auch Charcot-Fuß genannt, ist eine progressive, nicht-infektiöse Schwellung mit Überwärmung und Demineralisierung, gefolgt von Knochendestruktion und Deformierung, die in ca. $75 \%$ unilateral auftritt und in einer Defektheilung zum Stillstand kommt. Resultierende Fehlstellungen können zu neuropathischen Ulzera führen, die sich infizieren und Amputationen erforderlich machen kön- nen. Die häufigste, aber nicht einzige Ursache ist der Diabetes mellitus. Etwa $2 \%$ aller Diabetiker entwickeln einen CharcotFuß. Der pathophysiologische „Charcot-Prozess“ ist komplex, scheint aber untrennbar mit der vorausgehenden Neuropathie verbunden zu sein. Die C- und A $\delta$ - Fasern sind im Rahmen der diabetischen Neuropathie früh und häufig geschädigt, was ein Ungleichgewicht an CGRP, VIP, Substanz P und weiteren Transmittern erklärt. Störungen der Knocheninnervation verschieben das Verhältnis von Knochenan- und -abbau, von OPG und RANKL zugunsten des Abbaus. Demnach stellt die Fehlregulation nozizeptiver Nervenfasern auf molekularer Ebene eine pathophysiologische Brücke zwischen Diabetes mellitus und neurogener Inflammation dar.

\section{ABSTRACT}

The neuropathic osteo(arthro-)pathy, also called Charcot foot, constitutes a progressive, non-infectious, self-limiting, foot inflammation and demineralization followed by bone destruction and deformity, which occurs unilaterally in $75 \%$ of cases resulting in permanent defect of the foot involved. As a consequence of malposition, ulcers, potentially complicated by infections, can make amputations necessary. With about $2 \%$ of all diabetics affected, diabetes mellitus is the most frequent, but not exclusive cause of Charcot foot to date. The pathophysiological process leading to this condition is complex; it is, however, closely linked to a preceding neuropathy. The small $\mathrm{C}$ - und $\mathrm{A} \delta$ nerve fibers are particularly prone to damage in the early course of diabetic neuropathy leading to an imbalance of neurotransmitters such as CGRP, VIP, substance $P$, and others. Disturbances in bone innervation shift the equilibrium between bone formation and degradation represented by OPG and RAN$\mathrm{KL}$, respectively, in favor of demineralization. It is suspected that dysregulation of nociceptive nerve fibers builds the pathophysiological bridge between diabetes mellitus and neurogenic inflammation causing the formation of Charcot foot.

\section{Der Charcot-Fuß als Folge neuronaler Fehlregulation}

Der Begriff neuropathische Osteo-/Arthropathie oder auch Charcot-Fuß bezeichnet eine progressive, denervationsbedingte Inflam- mation und Demineralisation typischerweise eines, manchmal aber auch beider Füße mit der Gefahr schwerer Deformitäten bis hin zur Amputationsbedürftigkeit. Heute stellt die diabetische Neuropathie die bekannteste und gesundheitspolitisch wichtigste Ursache des Charcot-Fußes dar [1,2]. Etwa 2\% aller Diabetiker entwickeln 
einen solchen Charcot-Fuß [3, 4]. Die Tatsache, dass dieser aber auch bei erblichen Neuropathien wie z.B. der Transthyretin-assoziierten familiären amyloidotischen Polyneuropathie oder bei der Fehlbildungs- oder traumatisch bedingten Syringomyelie auftreten kann, leitet das Augenmerk auf die vielfältige Genese der Nervenschädigung.

Die Geschichte der Pathophysiologie zur Entstehung des Charcot-Fußes ist komplex. Schon im 18. Jahrhundert berichtete William Musgrave bereits über Nerven- und Gelenkschäden im Zusammenhang mit venerischen Erkrankungen [5]. Im 19. Jahrhundert beobachteten Mitchell sen. und jun. die Ausbildung schwerer sensomotorischer Defizite einhergehend mit Fußdeformitäten bei Patienten mit spinaler Tuberkulose und anderen Wirbelsäulenverletzungen [2]. Charcot selbst beschrieb die fortan als Charcot-Fuß geltende Osteoarthropathie bei Patienten mit Tabes dorsalis. Hier bestanden neben sensiblen Defiziten auch fortgeschrittene Paresen mit assoziierter Fehlstellung und Gelenkschäden.

Charakteristisch für den heutigen Charcot-Fuß ist im Frühstadium die nicht-infektiöse Schwellung mit Überwärmung. Im Weiteren kommt es zu Demineralisierung, gefolgt von Knochendestruktionen und bisweilen grotesker Deformierung, die in ca. $75 \%$ unilateral auftritt [6] und typischerweise in einem selbstlimitierenden Prozess in einer Defektheilung zum Stillstand kommt [4]. Die bisweilen erheblichen Fehlstellungen können zu neuropathischen UIzera führen, die zum Teil schwere Infektionen hervorrufen und letztlich Amputationen erforderlich machen können ( $\triangleright \mathbf{A b b}$. 1a, b, c, 2, 3).

Es ist bislang nicht geklärt, wodurch der „Charcot-Prozess“ ausgelöst wird. Unbestritten ist jedoch: Am Anfang der pathophysiologischen Ereigniskette steht die Nervenschädigung, oder: kein Charcot-Fuß ohne Neuropathie.

\section{Die diabetische Polyneuropathie - ein Überblick}

Der Diabetes mellitus ist die häufigste Ursache für erworbene Neuropathien in der westlichen Welt. Man geht davon aus, dass ca. die
Hälfte aller Menschen mit einem Diabetes mellitus eine klinisch manifeste Neuropathie entwickelt [7], wobei frühe Stadien der Erkrankung oftmals klinisch unbemerkt bzw. nur elektrophysiologisch nachweisbar verlaufen, sodass die Dunkelziffer wesentlich höher anzusiedeln sein dürfte [8,9].

Die Ursachen der diabetischen Neuropathie sind bis heute nicht in allen Details verstanden, jedoch finden sich Hinweise, dass es sich nicht um eine einzige Schadensursache handelt, sondern dass eine Vielzahl von Faktoren zu deren Entstehung beitragen. So finden sich multiple destabilisierte Stoffwechselwege [10], welche in ihrer Summe zu Zellschwellung, Mitochondriendysfunktion, Endoplasmatisches Retikulum (ER)-Stress, Inflammation und letztlich Apoptose führen.

Im Rahmen einer hyperglykämen Stoffwechsellage kommt es zur Akkumulation von Glukose in Nerven-, Nieren- und Retinazellen, wo unter Verbrauch von NADPH durch Reduktion der Zucker Sorbitol entsteht. Als zellschädigend wird einerseits die osmotische Wirkung des in der Zelle akkumulierenden Sorbitols diskutiert, zudem aber auch die fehlende Reduktionskapazität durch verminderte Verfügbarkeit von NADPH. Durch mitochondriale Dysfunktion und Bildung von Advanced-Glycation-End-Products (AGE) entsteht oxidativer Stress, sodass es zu DNA-Schäden und in der Konsequenz zur Zellnekrose sowie Zytokin-vermittelt (z.B. TNF- $\alpha$, IL-6) zur Inflammation kommt. Der Transkriptionsfaktor NFkB wird über den Phospholipase C Pathway exprimiert und in den Zellkern verlagert, was inflammatorische Prozesse verstärkt [10]. Die Überlastung des endoplasmatischen Retikulums („ER-Stress”) durch das Überangebot ungefalteter Proteine oder Störungen der Kalziumhomöostase kann ihrerseits zu pro-inflammatorischen Kaskaden führen [11]. Das Überangebot der glukoneogenen Aminosäure Alanin verleitet die Serin-Palmitoyl-CoA-Transferase, das geschwindigkeitsbestimmende Enzym der Sphingolipidsynthese, zum Substratwechsel, sodass durch vermehrte Verstoffwechselung von Alanin statt Serin die neurotoxischen Deoxy-Sphingolipide entstehen $[9,12]$.

Die diabetische Neuropathie kann sich fokal oder generalisiert präsentieren, dabei überwiegend die kleinen intraepidermalen C-
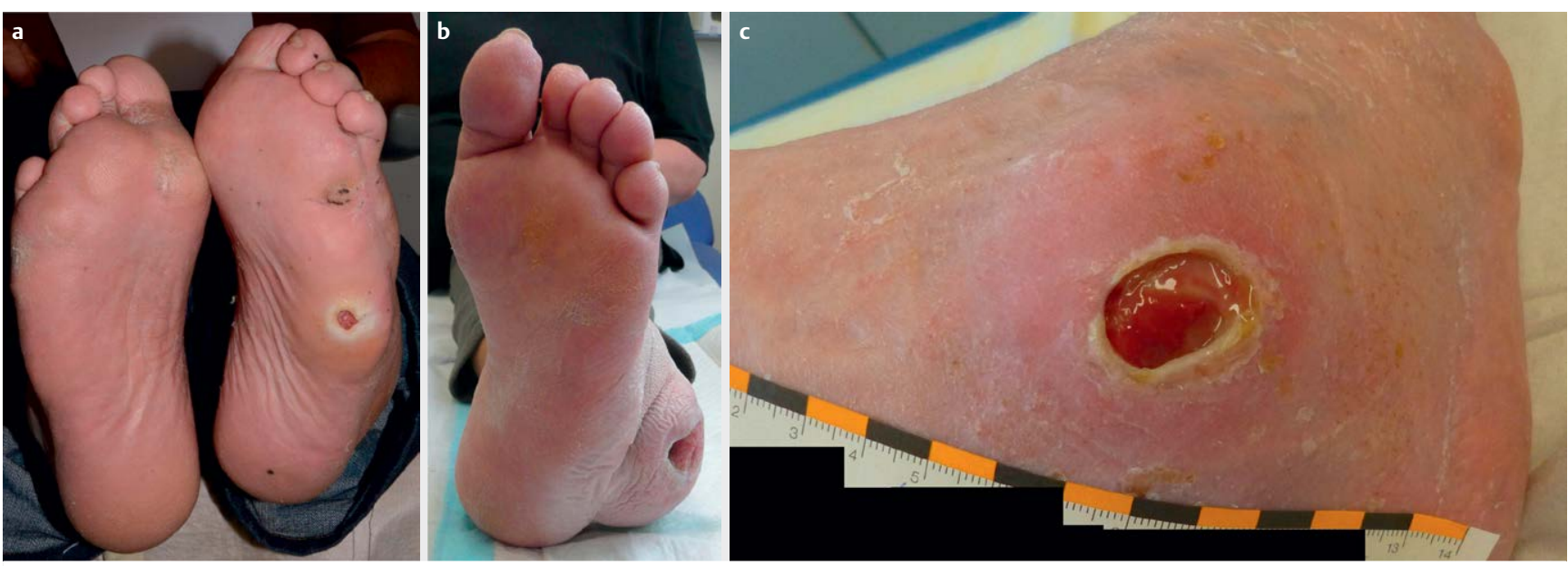

- Abb. 1 a-c Beispiele für verschiedene Stadien von Charcot-Füßen: a 59-jähriger Patient mit seit > 20 Jahren bestehendem Diabetes mellitus Typ 2 b Patient mit Hohlfuß und spontaner Weichteilschwellung c Ulkus am Außenknöchel eines bereits Vorfußamputierten Charcot-Fuß-Patienten mit langjährigem Diabetes mellitus. 


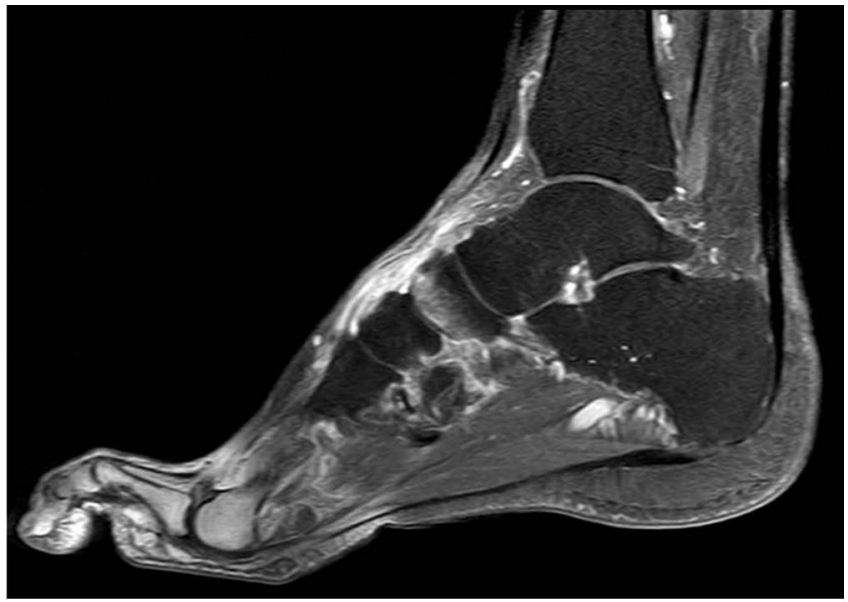

- Abb. $\mathbf{2}$ Die MRT des Patienten aus $>$ Abb. $\mathbf{1 b}$ zeigt in der T2-Wichtung ein intraossäres Ödem im distalen Anteil des Os naviculare, das erste Zeichen der neurogenen Osteoklastenstimulation. In Verbindung damit hat sich ein Ödem in den Weichteilen des Fußrückens ausgebildet. Die neurogenen Knochenödeme können - wie hier - in der Ausdehnung beschränkt, aber auch über mehrere Knochen ausgedehnt sein. Die Ödeme bilden sich unter Entlastung nach 2-3 Monaten zurück. Wenn der Fuß belastet wird, erfolgt eine Stimulation der Osteoklastentätigkeit mit entsprechendem Knochenabbau.

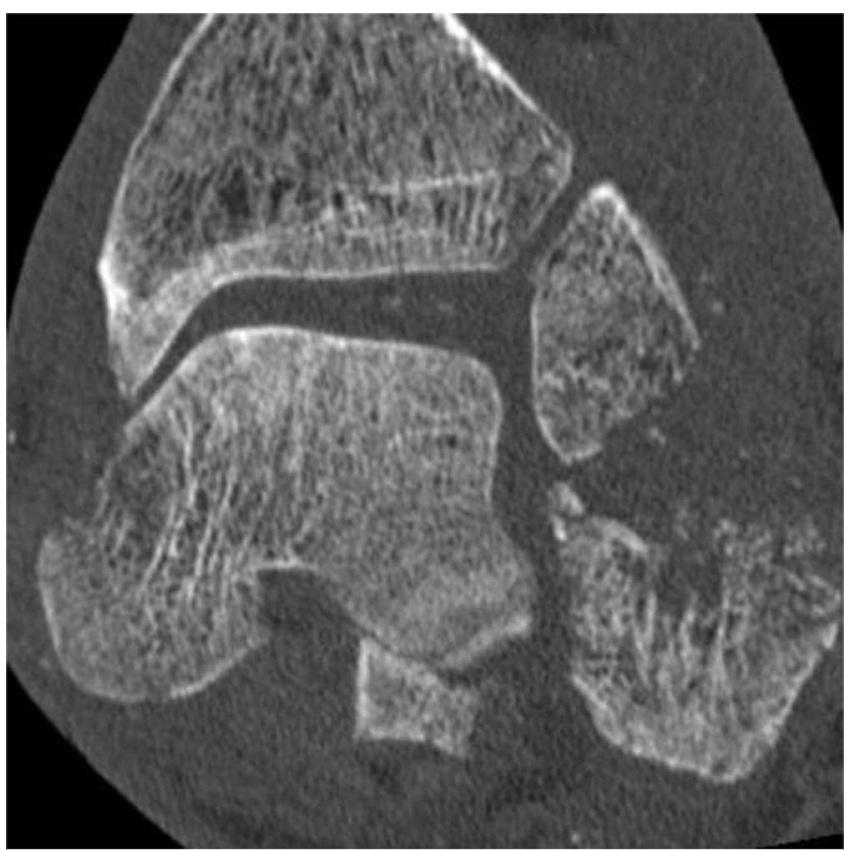

Abb. 3 Koronarer CT-Schnitt durch das Sprunggelenk eines 83-jährigen Patienten, bei dem der Fuß beim Gehen auf ebenem Boden unvermittelt nachgegeben hatte. Der Kalkaneus ist bis auf das Sustentaculum tali komplett nach lateral luxiert. Der Knochen ist deutlich hypomineralisiert, wahrscheinlich altersbedingt. Man erkennt multiple Lyseherde als Folge der neurogenen Ostoklastenstimulation. Am kranialen Kalkaneus sind die Lyseherde konfluiert und haben die Knochenoberfläche bis auf einen kleinen Rest an der Medialseite weitgehend beseitigt. Das Ausmaß der neurogenen Porosierung kann sehr unterschiedlich sein. Anders als in diesem Fall kann es sich auf eine begrenzte Region beschränken.

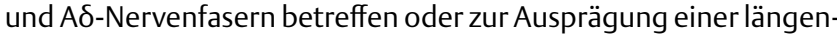
abhängigen Polyneuropathie führen. Die „typische“ und gleichzeitig am häufigsten anzutreffende Subform der diabetischen Neuropathie ist die distal symmetrische und somit längenabhängige diabetische Polyneuropathie, die etwa dreiviertel aller Subformen ausmacht [13]. Sensible Defizite, beginnend an den Füßen, stehen im Vordergrund, wobei das Berührungs- und Temperaturempfinden sowie die Spitz-Stumpf-Diskrimination besonders früh, im Verlauf aber auch die Pallästhesie und der Lagesinn betroffen sein können. Neuropathische Schmerzen, die v. a. als brennend oder stechend beschrieben werden, finden sich bei $25 \%$ der Patienten [14]. Demgegenüber bestehen Paresen bei 1-6\% aller Diabetiker, wobei diese wiederum v. a. die distale Muskulatur der Füße und Unterschenkel betreffen und in Kombination mit der afferenten sensiblen Störung die Sturzneigung dieser Patienten erhöhen [15]. Im späten Verlauf können Fußfehlstellungen und Störungen der Hauttrophik auftreten.

Eine weitere klinische Manifestationsform stellt die autonome diabetische Neuropathie dar, die selten isoliert, sondern wesentlich häufiger kombiniert mit anderen diabetischen Neuropathieformen auftritt. Das sympathische und/oder parasympathische System kann dabei betroffen sein, was durch gastrointestinale Passagestörungen, neurogene Blasenentleerungsstörungen, Erektions- und Ejakulationsstörungen, orthostatische Hypotonie, Ruhetachykardie, Störungen des Schwitzens sowie der Pupillomotorik manifest wird [16]. An der Haut führt die autonome Neuropathie zur Weitstellung der präkapillären Widerstandsgefäße mit der Folge einer Hyperämie. Andererseits bedingt diese eine Reduktion der Schweiß- und Talgsekretion, wodurch die Haut trocken und dadurch verletzlich wird.

Ebenfalls meist schmerzhafte Plexusneuropathien des Plexus lumbo-sacralis können vorkommen und werden dann als diabetische Amyotrophie (Bruns-Garland-Syndrom) bezeichnet [17]. Mononeuritiden können isoliert einen Hirn- (meist N. III, IV oder VI) oder Extremitätennerven oder einen thorakalen Nerven (Pseudohernie) betreffen.

\section{Klinisches Bild der Diabetischen Neuropathie}

Das charakteristische Bild stellen strumpfförmige Sensibilitätsstörungen, eine gestörte Hauttrophik sowie im späteren Verlauf auch Paresen, Fußfehlstellungen und die inneren Organe betreffende autonome Störungen dar. Die Empfindungsstörungen von Schmerz und Temperatur und die autonomen Beschwerden werden durch Schädigung der kleinen unmyelinisierten $\mathrm{C}$ - und $\mathrm{A} \delta$-Fasern verursacht, welche als besonders vulnerabel gelten und bei der diabetischen Neuropathie bereits in frühen Stadien betroffen sind [18].

Die diabetische Neuropathie kann verschiedene Verlaufsformen nehmen und ist somit klinisch oft nicht eindeutig von anderen, nichtdiabetischen Neuropathien zu unterscheiden. Bei der Untersuchung der (diabetischen) Polyneuropathie werden nach der klinischen Symptomatik drei funktionell wichtige Aspekte unterschieden:

1. Die sensible Neuropathie kann man orientierend durch Streichen mit dem Finger oder einem Wattebausch überprüfen. Es liegt nicht immer ein Sensibilitätsausfall vor, sondern häufig lediglich eine Sensibilitätsminderung. Ebenso gemindert kann die KaltWarm-, Spitz-Stumpf-, Positions- sowie die Vibrationsdiskrimination sein. Gleichzeitig treten häufig sensible Plus-Symptome in 
Form von kribbelnden (Parästhesien) und stechenden (Dysästhesien) Missempfindungen, eine Allodynie oder neuropathische Schmerzen auf, welche anamnestisch erfragt werden müssen.

2. Die motorische Neuropathie führt zu einer Schwächung und später zu einem Ausfall der Muskeltätigkeit, die meist von distal nach proximal fortschreitet. Die Schädigung beginnt an den Mm. interossei und den Mm. lumbricales [19], deren Funktion in der Beugung des Grund- und Streckung des Mittelgelenks besteht. Bei Ausfall ergibt sich eine Hammer-oder Krallenstellung der Zehen, da die Funktion der langen Zehenbeuger noch erhalten ist.

Im Verlauf können eine Fuß- und Zehenheberschwäche mit Steppergang und Sturzneigung und seltener auch proximale Paresen auftreten. Bei Befall der Hände führt die motorische Neuropathie oft zu Feinmotorikstörungen.

3. Die autonome Neuropathie hat Auswirkungen auf die inneren Organe wie Magen-Darm-, Herz-Kreislauf- und Urogenitaltrakt. An der Haut führt sie zur Weitstellung der präkapillären Widerstandsgefäße mit der Folge einer Hyperämie. Andererseits bedingt diese eine Reduktion der Schweiß- und Talgsekretion, wodurch die Haut trocken und dadurch verletzlich wird.

\section{Der Knochenmetabolismus}

Der Knochenstoffwechsel wird durch Zytokine beeinflusst bzw. gesteuert. Durch Aktivierung des Rezeptors RANK (Receptor Activator of NF-kB) wird der Transkriptionsfaktor NFkB (nuclear factor ,kappalight-chain-enhancer' of activated B-cells) in den Zellkern unreifer Osteoklastenprogenitoren verlagert, was die Differenzierung zu reifen Osteoklasten bewirkt. Als Agonist zu RANK wirkt der RANK-Ligand (RANK-L), während Osteoprotegerin (OPG) als Antagonist fungiert. Bei Patienten mit Charcot-Fuß ist das Verhältnis dieser beiden Faktoren, die RANKL/OPG Ratio, erhöht [20]. Als Ursache des Ungleichgewichtes zugunsten des Knochenabbaus wird das pro-inflammatorische Milieu und insbesondere die Überexpression von TNF- $\alpha$ angesehen $[4,21,22]$. Monozyten, die Vorstufen der Osteoklasten, erwiesen sich bei Patienten mit Charcot-Prozess als Apoptose-resistenter [22], was eine für die Akutphase spezifische Veränderung darstellt. Genetische Assoziationsstudien hypothetisieren zudem eine individuelle Suszeptibilität im Zusammenhang mit bestimmten Polymorphismen in den Genen OPG und RANKL [23].

\section{Periphere neuronale Regulation}

Die Innervation des Knochens findet sowohl durch myelinisierte als auch durch kleine unmyelinisierte Nervenfasern statt [24-26]. Mittels Histofluoreszenz konnten sowohl gefäßnah als auch in unmittelbarem Knochenkontakt Noradrenalin und vasoaktives intestinales Peptid (VIP) produzierende sympathische Nervenfasern dargestellt werden $[27,28]$. Nervenfasern sind sowohl im Periost, im Knochenkortex sowie im -mark vorhanden, in besonderer Dichte allerdings nah der osteochondralen Junktion im Bereich der Wachstumsfuge [29]. Nach Frakturen ist eine besonders hohe Nervenfaserdichte dort auszumachen, wo viel Knochenneubildung stattfindet [30], sodass die neuronale Regulation des Knochenmetabolismus nicht nur beim Knochenwachstum, sondern auch im Normalzustand zur Aufrechterhaltung des steady state und bei der Frakturheilung eine Rolle spielt. Nicht-synaptisch sezernierte Transmitter sind u.a. CGRP (calcitonin gene related peptide), Substanz P und VIP [26].
CGRP entsteht durch alternatives Splicing des Calcitonin-Gens. Wie auch Calcitonin selbst ist es durch Inhibition der Knochenresorption an der Kalziumregulation beteiligt, exprimiert wird CGRP allerdings nicht in den follikulären Schilddrüsenzellen, sondern in den knochennahen Nervenfasern. Capsaicin führt zur Destruktion dieser Zellen, was in einer verminderten Knochendichte resultiert $[29,30]$. Darüber hinaus ist CGRP dafür bekannt, überschießende zytokinvermittelte Inflammationsreaktionen zu begrenzen, indem es bspw. den TNF- $\alpha$ Spiegel senkt [31, 32].

Substanz $\mathrm{P}$ wird in Dorsalganglien produziert und durch Axonreflexe in terminalen Nervenendigungen freigesetzt. Als typischer Transmitter der neurogenen Inflammation ist es mit Vasodilatation und -extravasation, Glattmuskelkontraktion, Schmerzmodulation und Angiogenese assoziiert. Osteoklasten werden zur Ausreifung angeregt, der Effekt von RANKL potenziert und das RANKL/OPGVerhältnis zugunsten von RANKL verschoben [29, 33].

VIP reduziert die RANKL/OPG-Ratio durch Erhöhung zirkulierenden OPGs und hemmt die Sekretion pro-inflammatorischer Zytokine. Es bewirkt eine Osteoblastenaktivierung, erkennbar an der Expression von alkalischer Phosphatase, welche für den Knochenanbau zuständig ist, und führt zu einer gesteigerten Kalziumakkumulation in Knochennoduli und einer verminderten Osteoklastenformation [29].

Störungen der Knocheninnervation können also zu einem Ungleichgewicht von Neurotransmittern führen. Ein Mangel an CGRP und VIP verschiebt das Verhältnis von Knochenan- und -abbau, von OPG und RANKL zugunsten des Knochenabbaus. Zudem inhibiert CGRP die Produktion von proinflammatorischen Zytokinen wie TNF$\alpha$, was ebenfalls direkten Einfluss auf Osteoklastenprogenitoren hat. Ein lokaler CGRP Mangel könnte ein Auslöser (Trigger) für die Entwicklung des Charcot-Fußes nach heutigem Verständnis sein mit der Gefahr von Frakturierung, Fehlstellung, Ulzeration und Infektion. Letztere Komplikationen stellen selbst inflammatorische Reize dar, die den Prozess der Osteolyse verstärken und in Gang halten können.

\section{Zentrale Regulationsmechanismen}

Auch das zentrale Nervensystem übt Einfluss auf den Knochenstoffwechsel aus. Serotonerge Neurone der Raphe-Kerne aktivieren den ventromedialen Hypothalamus, welcher präganglionäre sympathische Neurone hemmt. Durch Reduktion des Sympathikotonus wird der Knochenaufbau gegenüber der -resorption gefördert. Leptin beeinflusst diesen Pathway negativ, wobei der genaue Mechanismus noch unverstanden ist. Auch bei der Appetitregulation sind Serotonin und Leptin Gegenspieler; und tatsächlich haben leptindefiziente Mäuse eine erhöhte Knochendichte [34]. Das anabol wirkende Serotonin wird zudem von Knochenzellen selbst produziert, gleichzeitig sind Serotoninrezeptoren im Knochen exprimiert, sodass Autoregulation als zusätzlicher serotonerger Mechanismus diskutiert wird [34].

\section{Nervenschädigung und neurogene Inflammation - die Brücke zum Knochenstoffwechsel}

Unter der neurogenen, nicht-infektiösen Inflammation versteht man die antidrome Freisetzung entzündungsfördernder Faktoren aus afferenten Nervenendigungen [35]. 
Entgegen ihrer eigentlichen Signalleitungsrichtung kommunizieren nozizeptive $C$ - und $A \delta$ - Fasern nicht-synaptisch mit Entzündungszellen, welche systemisch wirksame pro-inflammatorische Faktoren wie IL-2 und TNF- $\alpha$ sezernieren [36]. Die wichtigsten Transmitter neben Glutamat sind Neuropeptide wie Substanz P, VIP, CGRP, Hypophysen-Adenylatcyklase stimulierendes Peptid (PACAP) und Neuropeptid Y (NPY) [37].

Als nozizeptive Sensoren der C- und A $\delta$ - Fasern gelten unter anderem die Transient Receptor Potenzial (TRP) Kanäle, zu deren aktivierenden und sensitivierenden Faktoren Hitze oder Kälte, Protonen, reaktive Sauerstoffspezies, Stickstoffmonoxid sowie intrazelluläre Modulationswege zählen [38]. TRPV1-Kanäle aus C-Fasern sind gleichzeitig auch verantwortlich für die Peak-Vasodilatation nach Ischämie oder Stauung [39].

Die C- und A $\delta$ - Fasern sind im Rahmen der diabetischen Neuropathie früh und häufig geschädigt [18], was ein Ungleichgewicht an CGRP, VIP, Substanz P und weiteren Transmittern als Voraussetzung zum Erwerb des neuropathischen Fußsyndroms erklärt. Demnach stellt die Fehlregulation nozizeptiver Nervenfasern auf molekularer Ebene eine potentielle pathophysiologische Brücke zwischen Diabetes mellitus und neurogener Inflammation dar.

\section{Andere Knochenerkrankungen als Folge neuronaler Fehlregulation}

Das neuropathische Fußsyndrom ist nicht spezifisch für die diabetische Neuropathie. Die abhängig von der Ätiologie ungleiche Übernahme (sekundär) prophylaktischer Maßnahmen wie Fußpflege für Diabetiker durch die Kostenträger des Gesundheitssystems erschien schon in der Vergangenheit zweifelhaft und wurde folgerichtig ganz aktuell (Stand 03/2020) nach einer Gesetzesänderung auch auf andere Neuropathieformen ausgeweitet. Andere Neuropathien wie z.B. die hereditäre sensible und autonome Neuropathie (HSAN) oder die familiäre amyloidotische Polyneuropathie (FAP) können ebenfalls zu schmerzloser Überwärmung, Schwellung und Rötung mit Knochenabbau und Deformierung der Füße führen $[40,41]$. Die verminderte Schmerzwahrnehmung ist für alle diese 3 Erkrankungen charakteristisch.

Mit starken neuropathischen Schmerzen namensgebend assoziiert ist hingegen das chronische regionale Schmerzsyndrom (CRPS) [42]. Als Auslöser des CRPS wird charakteristischerweise eine Verletzung bzw. Operation der betroffenen Extremität beschrieben [43] Unabhängig davon, ob große Nervenfasern nachweislich geschädigt waren, konnte eine verminderte intraepider-

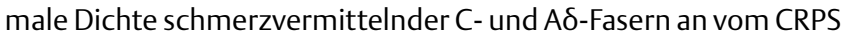
betroffenen Extremitäten hautbioptisch gezeigt werden [44, 45]. Es entsteht auch hier eine neurogene nicht-infektiöse Inflammation, vermittelt z.B. durch die Zytokine TNF- $\alpha$ und IL-1 $\beta[46,47]$. Pathophysiologisch bestehen darüber hinaus parallele Erklärungsansätze, die ebenfalls ein Ungleichgewicht des RANK-RANKL-OPGNFKB-Systems hypothetisieren [48, 49]. Die Gemeinsamkeit zwischen CRPS und Charcot-Fuß liegt somit in der nerval bedingten Inflammation und Abbau von Knochensubstanz. Im Unterschied zum Charcot-Fuß kann die Nervenschädigung beim CRPS reversibel sein, oft ist der Verlauf äußerst schmerzhaft, eine komplette Knochendestruktion ist beim CRPS nicht typisch, und auch der Weichteilschaden unterscheidet sich meist deutlich.

\section{FAZIT}

Ausgang der Pathogenese zur Entwicklung des CharcotFußes ist die neuropathiebedingte Osteopathie. Anders als bei den von Musgrave, Mitchell und Charcot beschriebenen Patienten steht beim heutigen Charcot-Fuß weniger die paresebedingte Fehlstellung als das kombinierte sensible und autonome Defizit im Vordergrund. Der Knochenmetabolismus wird neuronal reguliert. Wichtige Botenstoffe, die sich auf die Osteoblasten- und Osteoklastenreifung- und -aktivität modulierend auswirken sind CGRP, VIP und Substanz P, die aus knochennah gelegenen C- und Aס-Fasern sezerniert werden. Wenn diese Fasern (nicht nur) bei der diabetischen Neuropathie geschädigt werden, kann es zu einem unkontrollierten, überschießenden Knochenabbau kommen. Die initialen Skelettveränderungen gehen somit auf eine nervale Fehlregulation des Knochenstoffwechsels zurück, indem vorübergehend die knochenabbauenden Prozesse den Knochenanbau weit überwiegen. Im weiteren Verlauf können sich Frakturen, Fehlstellungen, Ulzerationen und Infektionen entwickeln. Für die Therapie und die Prognose ist es bei einem Neuropathiepatienten wichtig, bei jeder schmerzlosen Schwellung auch ohne vorausgegangenes Trauma und bei negativem Röntgenbefund v.a. an die neuropathische Osteopathie zu denken. Die Überaktivierung der Osteoklasten klingt nach acht bis 12 Wochen wieder ab, sodass die angemessene Therapie in der Entlastung für den entsprechenden Zeitraum besteht.

\section{Interessenkonflikt}

Die Autorinnen/Autoren geben an, dass kein Interessenkonflikt besteht.

Literatur

[1] Papanas N, Maltezos E. Etiology, pathophysiology and classifications of the diabetic Charcot foot. Diabetic foot \& ankle 2013; 4

[2] Trieb K. The Charcot foot: pathophysiology, diagnosis and classification. The bone \& joint journal 2016; 98-b: 1155-1159

[3] Nather A, Bee CS, Huak CY et al. Epidemiology of diabetic foot problems and predictive factors for limb loss. Journal of diabetes and its complications 2008; 22: 77-82

[4] Kaynak G, Birsel O, Guven MF et al. An overview of the Charcot foot pathophysiology. Diabetic foot \& ankle 2013; 4

[5] Kelly M. De Arthritide Symptomatica of William Musgrave (16571721): His Description of Neuropathic Arthritis. Bulletin of the history of medicine 1963; 37: 372-377

[6] Hartemann-Heurtier A, Van GH, Grimaldi A. The Charcot foot. Lancet (London, England) 2002; 360: 1776-1779

[7] Obrosova IG. Update on the pathogenesis of diabetic neuropathy. Current diabetes reports 2003; 3: 439-445

[8] Dyck PJ. Detection, characterization, and staging of polyneuropathy: assessed in diabetics. Muscle \& nerve 1988; 11: 21-32 
[9] Dohrn MF, Othman A, Hirshman SK et al. Elevation of plasma 1-deoxy-sphingolipids in type 2 diabetes mellitus: a susceptibility to neuropathy? European journal of neurology 2015; 22: 806-814, e855

[10] Sandireddy R, Yerra VG, Areti A et al. Neuroinflammation and oxidative stress in diabetic neuropathy: futuristic strategies based on these targets. International journal of endocrinology 2014; 2014: 674987

[11] Cameron NE. Role of endoplasmic reticulum stress in diabetic neuropathy. Diabetes 2013; 62: 696-697

[12] Bertea M, Rutti MF, Othman A et al. Deoxysphingoid bases as plasma markers in diabetes mellitus. Lipids in health and disease 2010; 9: 84

[13] Dyck PJ, Albers JW, Andersen $\mathrm{H}$ et al. Diabetic polyneuropathies: update on research definition, diagnostic criteria and estimation of severity. Diabetes/metabolism research and reviews 2011; 27: 620-628

[14] O'Connor AB. Neuropathic pain: quality-of-life impact, costs and cost effectiveness of therapy. PharmacoEconomics 2009; 27: 95-112

[15] Dyck PJ, Kratz KM, Karnes JL et al. The prevalence by staged severity of various types of diabetic neuropathy, retinopathy, and nephropathy in a population-based cohort: the Rochester Diabetic Neuropathy Study. Neurology 1993; 43: 817-824

[16] Haensch CA, Luhrs A. Autonomic Disorders (Reprinted). Klinische Neurophysiologie 2018; 49: 161-164

[17] Barohn RJ, Sahenk Z, Warmolts JR et al. The Bruns-Garland syndrome (diabetic amyotrophy). Revisited 100 years later. Archives of neurology 1991; 48: 1130-1135

[18] Umapathi T, Tan WL, Loke SC et al. Intraepidermal nerve fiber density as a marker of early diabetic neuropathy. Muscle \& nerve 2007; 35 : 591-598

[19] Severinsen K, Andersen H. Evaluation of atrophy of foot muscles in diabetic neuropathy - a comparative study of nerve conduction studies and ultrasonography. Clinical neurophysiology: official journal of the International Federation of Clinical Neurophysiology 2007; 118: 2172-2175

[20] Ndip A, Williams A, Jude EB et al. The RANKL/RANK/OPG signaling pathway mediates medial arterial calcification in diabetic Charcot neuroarthropathy. Diabetes 2011; 60: 2187-2196

[21] Jeffcoate W]. Charcot neuro-osteoarthropathy. Diabetes/metabolism research and reviews 2008; 24 (Suppl 1): S62-S65

[22] Uccioli L, Sinistro A, Almerighi C et al. Proinflammatory modulation of the surface and cytokine phenotype of monocytes in patients with acute Charcot foot. Diabetes care 2010; 33: 350-355

[23] Bruhn-Olszewska B, Korzon-Burakowska A, Wegrzyn G et al. Prevalence of polymorphisms in OPG, RANKL and RANK as potential markers for Charcot arthropathy development. Scientific reports 2017; 7: 501

[24] Serre CM, Farlay D, Delmas PD et al. Evidence for a dense and intimate innervation of the bone tissue, including glutamate-containing fibers. Bone 1999; 25: 623-629

[25] Chenu C. Role of innervation in the control of bone remodeling. Journal of musculoskeletal \& neuronal interactions 2004; 4: 132-134

[26] Jones KB, Mollano AV, Morcuende JA et al. Bone and brain: a review of neural, hormonal, and musculoskeletal connections. The lowa orthopaedic journal 2004; 24: 123-132

[27] Duncan CP, Shim SS J. Edouard Samson Address: the autonomic nerve supply of bone. An experimental study of the intraosseous adrenergic nervi vasorum in the rabbit. The Journal of bone and joint surgery British volume 1977; 59: 323-330

[28] Hohmann EL, Elde RP, Rysavy JA et al. Innervation of periosteum and bone by sympathetic vasoactive intestinal peptide-containing nerve fibers. Science (New York, NY) 1986; 232: 868-871

[29] Lerner UH, Persson E. Osteotropic effects by the neuropeptides calcitonin gene-related peptide, substance $P$ and vasoactive intestinal peptide. Journal of musculoskeletal \& neuronal interactions 2008; 8: 154-165
[30] Li ], Kreicbergs A, Bergstrom J et al. Site-specific CGRP innervation coincides with bone formation during fracture healing and modeling: A study in rat angulated tibia. Journal of orthopaedic research: official publication of the Orthopaedic Research Society 2007; 25: 1204-1212

[31] Gomes RN, Castro-Faria-Neto HC, Bozza PT et al. Calcitonin generelated peptide inhibits local acute inflammation and protects mice against lethal endotoxemia. Shock (Augusta, Ga) 2005; 24: 590-594

[32] Chiu IM, Heesters BA, Ghasemlou N et al. Bacteria activate sensory neurons that modulate pain and inflammation. Nature 2013; 501: 52-57

[33] Sohn SJ. Substance P upregulates osteoclastogenesis by activating nuclear factor kappa B in osteoclast precursors. Acta oto-laryngologica 2005; 125: 130-133

[34] Lavoie B, Lian JB, Mawe GM. Regulation of Bone Metabolism by Serotonin. Advances in experimental medicine and biology 2017; 1033: 35-46

[35] Richardson JD, Vasko MR. Cellular mechanisms of neurogenic inflammation. The Journal of pharmacology and experimental therapeutics 2002; 302: 839-845

[36] Chiu IM, von Hehn CA, Woolf CJ. Neurogenic inflammation and the peripheral nervous system in host defense and immunopathology. Nature neuroscience 2012; 15: 1063-1067

[37] Sorkin LS, Eddinger KA, Woller SA et al. Origins of antidromic activity in sensory afferent fibers and neurogenic inflammation. Seminars in immunopathology 2018; 40: 237-247

[38] Gouin O, L'Herondelle K, Lebonvallet $\mathrm{N}$ et al. TRPV1 and TRPA1 in cutaneous neurogenic and chronic inflammation: pro-inflammatory response induced by their activation and their sensitization. Protein \& cell 2017; 8: 644-661

[39] Marche P, Dubois S, Abraham P et al. Neurovascular microcirculatory vasodilation mediated by $\mathrm{C}$-fibers and Transient receptor potential vanilloid-type-1 channels (TRPV 1 ) is impaired in type 1 diabetes. Scientific reports 2017; 7: 44322

[40] Benson MD. The hereditary amyloidoses. Best practice \& research Clinical rheumatology 2003; 17: 909-927

[41] Feldman DS, Ruchelsman DE, Spencer DB et al. Peripheral arthropathy in hereditary sensory and autonomic neuropathy types III and IV. Journal of pediatric orthopedics 2009; 29: 91-97

[42] Marinus J, Moseley GL, Birklein F et al. Clinical features and pathophysiology of complex regional pain syndrome. The Lancet Neurology 2011; 10: 637-648

[43] Harden RN, Bruehl S, Galer BS et al. Complex regional pain syndrome: are the IASP diagnostic criteria valid and sufficiently comprehensive? Pain 1999; 83: 211-219

[44] Oaklander AL, Rissmiller JG, Gelman LB et al. Evidence of focal small-fiber axonal degeneration in complex regional pain syndrome-I (reflex sympathetic dystrophy). Pain 2006; 120: 235-243

[45] Albrecht P], Hines S, Eisenberg $E$ et al. Pathologic alterations of cutaneous innervation and vasculature in affected limbs from patients with complex regional pain syndrome. Pain 2006; 120: 244-266

[46] Birklein F. Complex regional pain syndrome. Journal of neurology 2005; 252: 131-138

[47] Bruehl S. An update on the pathophysiology of complex regional pain syndrome. Anesthesiology 2010; 113: 713-725

[48] de Mos M, Laferriere A, Millecamps M et al. Role of NFkappaB in an animal model of complex regional pain syndrome-type I (CRPS-I). The journal of pain : official journal of the American Pain Society 2009; 10: 1161-1169

[49] Kramer HH, Hofbauer LC, Szalay G et al. Osteoprotegerin: a new biomarker for impaired bone metabolism in complex regional pain syndrome? Pain 2014; 155: 889-895 